DOI: $10.35643 /$ Info.26.1.16

Reseña

\title{
Mirar la Universidad desde la extensión
}

Prtisch, F., Verrua, R., Pintado, X. \& Varela, J. (2020). Miradas sobre la Extensión desde los EFI. La construcción de la extensión la experiencia de los EFI de la FIC: emergentes hacia una formación integral. Montevideo: Facultad de Información y Comunicación. Universidad de la República.

\section{Carlos Santos $^{\mathrm{a}}$}

a Centro Universitario Regional del Este / Facultad de Ciencias Sociales - Universidad de la República, Profesor Adjunto, Uruguay. ORCID: 0000-0002-1632-8129. Correo electrónico: carlos.santos@cure.edu.uy.

\section{Abrir}

Cuando en octubre de 2009 se inició el camino hacia la generalización de los Espacios de Formación Integral en la Universidad de la República se partió de una amplia y diversa trayectoria de trabajo en extensión en la institución. Una de esas experiencias, mencionadas explícitamente en la resolución del Consejo Directivo Central, era la desplegada por el área de Comunicación Comunitaria de la Licenciatura en Ciencias de la Comunicación. Es llamativo que en el texto Miradas sobre la extensión desde los EFI, sus autores Federico Pritsch, Romina Verrua, Ximena Pintado y Judith Varela se paren desde ese momento al presente para identificar elementos relevantes en el proceso hacia una formación integral.

Ya ha transcurrido más de una década y los Efi se han constituido en una herramienta indiscutible para incorporar la extensión en la currícula y de esa manera también contribuir a la renovación de la enseñanza.

Este libro nos presenta una oportunidad única de profundizar en las características de estos espacios, en las valoraciones de sus participantes y en unas claves analíticas que seguramente serán muy valiosas para la evaluación de esta política universitaria y su proyección, no sólo en el ámbito de la Facultad de la 
Información y la Comunicación (FIC) sino en toda la Universidad de la República.

\section{Mirar}

Los ejercicios analíticos fundados en investigaciones sistemáticas sobre la política de la integralidad no abundan en la Universidad de la República. Sólo por ese hecho la edición de Miradas sobre la extensión desde los EFI es un hecho saludable. Pero lo es más cuando arroja una visión en profundidad para la FIC en un período de importantes transformaciones (2013-2017) marcado por la aplicación de la nueva Ordenanza de Grado de la Universidad de la República, la puesta en marcha de los nuevos planes de estudio de sus tres carreras y el inicio de una nueva vida institucional -materializada en la mudanza a nuevo edificio común- en la que conviven las trayectorias institucionales previas de sus dos institutos constitutivos (la Escuela Universitaria de Bibliotecología y Archivología en el Instituto de Información y la Licenciatura en Ciencias de la Comunicación en el Instituto de Comunicación).

En términos de trayectorias también es relevante señalar aquí la propia trayectoria del equipo autoral del texto, entre quienes incluyo a la prologuista Siboney Moreira, bajo cuya coordinación en la Unidad de Desarrollo de la Extensión y las Actividades en el Medio (UDEA) surgió la iniciativa de realizar una sistematización de los Efi de la FIC. Son importantes las trayectorias de estas personas al punto que vienen al texto en varias oportunidades, porque dan cuenta de la relevancia que tiene la incorporación de la extensión como actividad estudiantil, ya que el pasaje por ellas marca a las personas de una manera tal que se generan compromisos con esta función que la convierten en mucho más que eso: la extensión pasa a ser una perspectiva desde la cual mirar a la totalidad de nuestra vida universitaria. De la experiencia estudiantil a la participación en proyectos estudiantiles, a desempeñarse como becarios hasta trabajar como docentes en diferentes estructuras de la facultad, la extensión permea las experiencias y los sentidos de esas trayectorias. Por ello es que decimos que estas Miradas sobre la extensión desde los EFI también constituyen una mirada a la Universidad, y en particular a la FIC desde la extensión. 


\section{Hablar}

En tiempos en que la Universidad de la República se encuentra implementando un nuevo Estatuto del Personal Docente y surgen preguntas sobre el papel de las estructuras de apoyo a las funciones universitarias (tanto a nivel central como a nivel de cada servicio universitario) este libro es un documento que demuestra el carácter fundamental de las estructuras de apoyo, en particular en este caso, a la extensión universitaria. ¿Desde dónde podrían generarse procesos sistemáticos de análisis, reflexión y procesamiento de información sobre la extensión y la integralidad? ¿De qué otro modo se podrían orientar las políticas universitarias sino es sobre la evidencia que ordenan y caracterizan estos equipos? Una parte central del material analizado en el libro es producto de los Planes de Trabajo y sus respectivas Evaluaciones que anualmente los servicios universitarios remiten a la Comisión Sectorial de Extensión y Actividades en el Medio. Lejos de tener un sentido burocrático, Federico, Romina, Ximena y Judith demuestran la vitalidad de esos números y proporciones al hacerlas hablar con la experiencia de docentes que integran equipos de los Efi. Las entrevistas en profundidad desplegadas sobre 8 de estos Efi echan luz sobre algunas de los elementos no definidos o definidos superficialmente en los formularios presentados por 62 equipos de trabajo de Efi desarrollados entre 2013 y 2017. Sin dudas es invaluable contar con esta información disponible en cada uno de los servicios universitarios, pudiendo establecer líneas de mediana duración en términos de la participación docente y estudiantil, el tipo de vínculo con actores sociales, la articulación con programas plataforma y las consideraciones acerca de la construcción de interdisciplina que realizan los propios equipos. También queda en evidencia en el trabajo la relevancia de la articulación de la Red de Extensión, espacio de construcción permanente entre todos los servicios universitarios y los programas plataforma que se evidencia en la adaptación de algunas dimensiones de la matriz de evaluación desarrollada en el el trabajo de la Red para pensar las particularidades de la propia experiencia de la FIC.

\section{Pensar}


Para quienes hemos tenido la fortuna de conocer de cerca el proceso de implementación de la política de extensión e integralidad en la Universidad de la República en la última década, sin duda los elementos más atractivos de Miradas sobre la extensión desde los EFI tienen que ver con el análisis de la información sistematizada en esta escala de mediana duración y con las valoraciones de los participantes directos de cada una de las experiencias. Un primer dato relevante tiene que ver con la temporalidad. Como plantea la Ordenanza de Grado de la Universidad de la República y los propios nuevos planes de estudio de las tres carreras de la FIC las unidades curriculares están organizadas semestralmente. Sin embargo una proporción importante de los Efi analizados tienen una duración anual. Esto tiene para los autores una doble lectura: la necesidad de ajustar los tiempos institucionales a los tiempos de los colectivos sociales con quienes se interactúa, por un lado, y la complejidad de los procesos interdisciplinarios e integrales desde el punto de vista formativo. Podría decirse que para una parte importante de los actores protagonistas de estas acciones integrales el formato que emerge del nuevo marco curricular no es suficiente para el despliegue de la potencia integral. Habrá aquí, quizás, una nota a tomar a la hora de revisar los planes de estudios y sus temporalidades.

Por otro lado hay una novedosa proposición de los autores al considerar etapas en la participación de los diferentes actores involucrados en los Efi. Es así que plantean una etapa de construcción de demanda (orientada principalmente al vínculo con actores sociales), una de implementación (a la que se suman los estudiantes) y una de evaluación/reflexión (que, con diferentes intensidades según cada uno de los casos, involucra a todos los participantes). Como queda en evidencia, la clave en estas etapas es el sostenimiento del proceso tanto desde el punto de vista social como desde el punto de vista del equipo universitario. El trabajo permite aproximarnos a diferentes estrategias, que tienen mucho de flexibilidad y trabajo artesanal para asegurar esta dimensión del trabajo integral. Quizás de los elementos que deje más lecturas abiertas del texto tiene que ver con la interdisciplina en el marco de la integral. Un dato contundente que aporta Miradas sobre la extensión desde los EFI es que la mayor parte de la vinculación con otras disciplinas se da dentro del área Social y Artística de la Universidad de 
la República, si bien se mencionan otras vinculaciones (como Geografía, Arquitectura o Agronomía). Ahí quedan seguramente muchas de las preguntas sobre las cuales partir a partir de este trabajo para profundizar las reflexiones.

\section{Cerrar}

Miradas sobre la extensión desde los EFI es una lectura ineludible para revisar la experiencia de curricularización de la extensión y la renovación de la enseñanza en la Universidad de la República. En particular arroja elementos novedosos de análisis a partir de sistematización de información y realización de entrevistas que lo coloca como un producto relevante para pensar la proyección de la política de los Espacios de Formación Integral no sólo en la FIC sino en toda la Universidad de la República. Un trabajo serio, comprometido y sistemático al cual habrá que tomar en cuenta para estar a la altura del trabajo no sólo de sus autores sino de todos los docentes, estudiantes y colectivos sociales involucrados en los procesos que estudia.

\section{Referencias bibliográficas}

Prtisch, F., Verrua, R., Pintado, X. \& Varela, J. (2020). Miradas sobre la Extensión desde los EFI. La construcción de la extensión la experiencia de los EFI de la FIC: emergentes hacia una formación integral. Montevideo: Facultad de Información y Comunicación. Universidad de la República. 